Original Research Paper

\title{
Inter Cluster Group Management in Wireless Sensor Networks
}

\author{
${ }^{1}$ R. Rohini, ${ }^{2}$ Adamu Murtala Zungeru, ${ }^{3}$ S. Ravi and ${ }^{4}$ Dasari Narasimha Rao \\ ${ }^{I}$ Computer Science and Engineering, Vivekanandha College of Engineering for Women, Namakkal, Tamilnadu, India \\ ${ }^{2,3}$ Department of Electrical, Computer and Telecommunications Engineering, \\ Botswana International University of Science and Technology, Palapye, Botswana \\ ${ }^{4}$ Department of Electrical Power Engineering, Defense University, Debrezeit, Ethiopia
}

\author{
Article history \\ Received: 13-01-2018 \\ Revised: 25-02-2018 \\ Accepted: 19-04-2018 \\ Corresponding Auhor: \\ Adamu Murtala Zungeru \\ Department of Electrical, \\ Computer and \\ Telecommunications \\ Engineering, Botswana \\ International University of \\ Science and Technology, \\ Palapye, Botswana \\ Email: zungerum@biust.ac.bw
}

\begin{abstract}
Wireless Sensor Networks (WSNs), an emerging large-scale network system can be embedded for any application. The designers of wireless sensor network have to consider the quality of service agreements from a user perspective. The WSN is very rapid in dynamic changing topology and has less energy, computing and communication resources. The network design has to fulfill the QoS parameters like reliability, timeliness and lifetime of the network. In wireless sensor network, when two nodes transmit messages through a common visible node and collision occurs between these nodes is refers to as Hidden Node Problem. Many researchers have reviewed the Hidden node problem affecting the throughput and lifetime of the network. This paper proposed Inter-Cluster Grouping Mechanism (ICGM) to handles the problem of inter-cluster grouping and hidden node for the improvement of Quality of Service (QoS) factors in WSN. The performance of WSN degrades due to the occurrence of inter-cluster interference caused by different grouping mechanism as imposed in different clusters. The proposed mechanism (ICGM) overcome the problem of inter-cluster interference and increases the QoS in WSNs.
\end{abstract}

Keywords: Wireless Sensor Network, Hidden Node, Inter-Cluster Interference, Quality of Service, Inter-Cluster Group Mechanism, Throughput, Reliability

\section{Introduction}

Wireless sensor network is an emerging field of the wireless communication and can be adapted towards fields, rescue operations, organizations, etc. The wireless sensor networks have a set of sensor nodes which will be moving in any direction and an unmovable base station which coordinates the signals sent and received from the sensor nodes. The sensor node has different components like an antenna, battery and sensor module. The sensor module measures environmental parameters that surround the sensor and transforms the ambient energy into electric signals. Information on events that are happening within the vicinity of the sensor is obtained through processing of the information by the processor module and the data is transmitted via radio transmitter to a sink or destination node. A wireless communication network is formed in an ad hoc manner where sensor nodes are able to independently organize themselves with no proper coordination. This is found in most WSNs applications. The source of power for the sensor nodes is battery, which is usually not re-chargeable or replaceable especially when the sensor nodes are expected to operate with no human intervention for longer periods of time during the application (Koubaa, 2009). Careful resource management is therefore a prime concern in the design of wireless sensor networks. It can be achieved through energy saving techniques such as Radio Optimization, Data Reduction, Sleep/Wakeup methods, Energy Efficient routing protocols and Energy Harvesting. Technological advances have led to reduced size and cost of the sensors and hence have fueled interest in the possible use of a large set of disposable unattended sensors. Among those, the battery is an essential component, which decides the lifetime of the sensor node and the network. As such, the energy available for the sensor node has to be utilized efficiently to meet the quality of service parameters. Wireless sensor networks performance can be improved upon by careful study in the area of mitigation of hidden node collision (Sheu et al., 2009; Tobagi and Kleinrock, 2009; Farahani, 2008; Pesovic, 2010). The WSNs has gained 
lot of application areas, as such, real deployment of the network need to be investgated using some siumation software before implementation as can be found in (Jurcik, 2007).

The wireless sensor nodes use communication protocols like IEEE 802.1e or Zigbee for transferring data packets between them. When two nodes participate in a transmission through a known intermediate node whereby the source and destination node does not become visible to each other, then there will be a collision, and it is referred to as "Hidden Node Problem." To handle this collision problem, there are different approaches been prescribed; HNam (Koubaa, 2009), is one among the method for the Hidden node problem. In HNam, an inter-cluster grouping mechanism is described to avoid hidden node problem.

The grouping mechanism used varies between clusters so that the protocol enforced for a Contention Access Period (CAP) of any CSMA based protocol will be varying at all the time. We cannot be sure about the grouping mechanism enforced at other clusters and the nodes under the cluster. This generates the inter clusters interference for the transmission of the packets.

However, conventional Wireless sensor networks uses contention-based medium access control (MAC) protocols, which is a family of Carrier Sense Multiple Access(CSMA). One of the drawback of CSMA is that, it's perfromance degrades significantly whenever the nodes in the network are increased, which inherently increases the traffic load, hence, leads to the growing of message collissions in the network. The performance degradation get worsen due to the impact of the Intercluster Interference, which is caused by different grouping mechanism and Contention Access Period (CAP). Inter Cluster Interference (ICI) affect four QoS metrics such as:

- Throughput: This represents the amount of traffic effectively received by a cluster head and reduced due to ICI based on asynchronous CAP and grouping mechanism

- Transfer delay: This is a function of the time taken to get to the destination node. A message may encounter delay due to retransmission base on ICi as it originates from source node towards the destination node

- Energy-efficiency: This is a ratio of the the product of success rate of packets by the total packets that originates from the source nodes over the total energy consumed

in

the network

$\left(\right.$ Energyefficiency $\left.=\left(\frac{\text { Success rate } * \text { Total packet sent tothe sink }}{\text { Total energy consumed }}\right)\right)$.

The energy efficiency decreases as each collision causes a new retransmission.
- Reliability: This is the ability of messages guaranteed to reach the destination nodes in complete and uncorrupted and in the order they were sent can be affected, since many applications can abandon message transmission following amount of retransmissions

From the literature it is clear that no efficient method has proposed for the identified problem. We propose a new grouping mechanism refers to as Inter-Cluster Grouping Mechanism (ICGM) to handle the Inter cluster interference problem.

To the best of our knowledge, the proposed mechanism (ICGM) is the first work that concentrate on these challenges and present useful solution to all of them. ICGM as the proposed mechanism proved the strength of the protocol through extensive simulation.

In this paper, we were able to address and make the following contribution to knwoledge:

- Inter-cluster Grouping Mechanism is an easy and well-organized mechanism for solving the problem of Inter-cluster Interference in coordinated single or multiple cluster based WSNs on node grouping approach. This mechanism iillustrates that ICGM is very easy to implement, in dissimilarity to the grouping mechanism

- This mechanism shows how to incorporate ICGM in the IEEE 802.15.4/ZigBee protocols with minor add-ons and ensuring backward compatibility with the default specifications

- The performance of the ICGM mechanism is evaluated through an experimental test-bed, showing significant QoS improvements

The rest of the paper is organized as follows: Section 2 presents the related works on the detection methodology of hidden nodes in the wireless sensor networks, while Section 3 presents the proposed Inter cluster Grouping mechanism. Section 4 presents the results and discussion and the paper is concluded in Section 5.

\section{Related Works}

The hidden-node problem and Intercluster interference are known to be a severe source of performance degradation in wireless communication networks. Shrestha et al. (2013; Poonguzhali, 2012), a mathematical analysis is derived based on queueingtheory and count the collision of the hiddennode problem on the act of small-scale linear wireless networks. A lot of works have concentrate on solutions for removing or dropping the crash of the hidden-node problem in wireless networks and it is categorized as: (1) Busy tone mechanisms; (2) Request- To-Send/Clear-To- 
Send (RTS/CTS) mechanisms; (3) Carrier-sense tuning mechanisms; (4) Interference cancellation mechanisms and (5) Node grouping mechanisms.

HNAM (Koubaa, 2009), propose a node grouping mechanism to handle the hidden node problem. It uses the Request/Response message for the node grouping mechanism where the node which wants to join in a cluster sends a group joins request. The cluster head has the bidirectional connection with other nodes in the network so that all the nodes will receive the group join request in the cluster. However, only the cluster head sends the acknowledgment for the group join request. On receiving the acknowledgment the new node starts the three-way handshake method to join in the cluster, and other nodes in the cluster also join the group. In this mechanism, the cluster head allocates the contention access period for each group to maximize the lifetime of the network. Based on the contention access period, the nodes in a group starts transmission to avoid a collision which generates hidden node problem.

Shrestha et al. (2013; Poonguzhali, 2012; Ranjani, 2012; Mehta, 2012; Hajiaghajani, 2012; Koscielnik, 2009) the concept of hidden node collision which is CSMA/CA-based multi-hop wireless sensor networks was introduced, and this is with respect to scheduling concept in addidtion to network planning model to alleviate this problem. The papers also address issues that relates to energy efficient relaization of clustering patch routing protocols based WSN, in addition to multitarget tracking, throughput and C-Leach were disccused in detailed by the authors.

In a related work, authors in (Yang and Huang, 2008; Ghosh and Basagni, 2008) proposed QoS routing which is based on link stability using dynamic delay prediction and the mitigation of the impact of node mobility in Adhoc network.

Rohini and Ravi (2017), a simple and efficient methodology has been proposed for clustering the nodes based on node weighting Algorithm. This algorithm discover the weight of all adjacent node of the cluster head and get the Euclidean distance from cluster head to all of its adjacent nodes within the cluster. The performance of the proposed system is analyzed in terms of energy consumption, packet delivery ratio and latency against the following attacks: Packet dropping attack, false reporter attack and acknowledgment hacking attack.

Rohini and Gnanamurthy (2016), a novel clustering mechanism is described for identifying the hidden nodes. The work described that, hidden nodes may lead to degredation in network performance. The performance in question includes throughput, energy efficiency, reliability and latency. The paper further shows that, energy consumption of the nodes in the network can be minimized and hidden nodes problem in the network can be solved through the detection of hidden nodes in the network of wireless sensors. The algorithm shows minimal performance using the metrics highlighted.

Thampi et al. (2016), static Clustering and adaptive Fractional Frequency Reuse (CFFR) is proposed as means of practical and scalable solution to mitigating interference on the uplink of a network. The authors derived an analytical capacity equations which are based on Zero Forcing (ZF) and Linear Minimum Mean Squared Error (LMMSE) receivers. However, the methods shows that, inter-cluster interference in the network degrades the performance of the network, even for the smallest interference from the neighbouring clusters. The authors also shows that, CFFR algorithm is complex as compared to DC in terms of running time.

However, there is still issues in the network with respect to quality of service of the network. Hence, we proposed Inter-Cluster Grouping Mechanism (ICGM) to handles the problem of inter-cluster grouping and hidden node for the improvement of Quality of Service (QoS) factors in WSN.

\section{The ICGM Mechanism}

\section{System Model}

This work considered a wireless network of multiple cluster for which there exist atleast a node in each cluster with bidirectional radio connectivity and having the bidirectional connectivity with all of the other cluster nodes in the network as seen in Fig. 1. The node with the bidirectional radio connectivity with all other nodes in the network is refered to as the Cluster Head $(\mathrm{CH})$. The main duty of the cluster head is to cordinate other nodes in the cluster and also, atleast support routing capabilities, and ensuring reliable communication between nodes in the same cluster. For this system, it is assumed that nodes are to contend for medium access for any time Contenetion Access Period (CAP) is needed using a contention based MAC.

\section{Inter-Cluster Grouping}

Initially, each cluster head $\mathrm{CH}$ broadcast cluster group join request message to its neighbors with the assumption that the intracluster grouping is already done. Each cluster head within the transmission range of the transmitter will receive the message and generate an acknowledgment to confirm the bi-directional connection present between them. The sender will keep track of a set of cluster heads which has acknowledged the cluster join request message. 


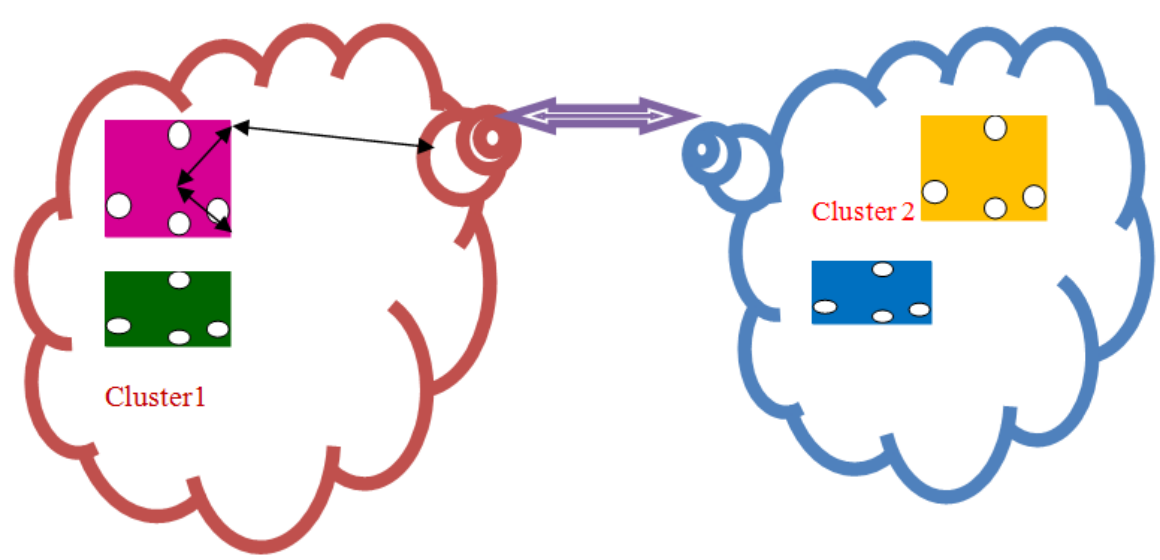

Fig. 1: A system model of inter cluster grouping

\section{Step 1: Cluster Join Request}

The cluster head broadcasts the cluster join request with the cluster address in the destination field. Let us consider cluster head $\mathrm{CH}_{\mathrm{i}}$ sends cluster to join request to the neighbor Cluster head $\mathrm{CH}_{\mathrm{j}}$ using a specific broadcast address specifying the group address in the destination address field. CM is defined as the inter-cluster group management address of the cluster, which must be acknowledged by the $\mathrm{CH}_{\mathrm{n}}$. The Acknowledgment message (ACK) will be received by all clusterheads with which it has bi-directional links.

The neighboring cluster heads send back the acknowledgment notifying $\mathrm{CH}_{\mathrm{i}}$ that about the correct reception of the Cluster join request. At the end of this procedure, each cluster head will know about the available neighboring cluster heads and their group address for broadcasting. After receiving the acknowledgment, it sends a confirmation message that it has selected the set of cluster heads as its neighbors for further transmission.

\section{Step 2: Inter Cluster Interference Handling}

The cluster head allocates the time slot for the Contention access period for each group of the cluster. The synchronization of CAP helps to assign and control the groups in participating in different data communication. The data transfer between two different cluster nodes is controlled by the cluster heads as follows.

\section{Group Controlling}

The cluster head broadcasts a control message which specifies the new data transfer into the cluster using NPMessage. The packet (message) will then be recovered by all the participating nodes in the cluster from different groups. The broadcast message has the destination field which has the group address and unique node address of the ultimate destination. Upon receiving this message, the nodes present in the same group respond to the message by sending an acknowledgment, which specifies that they are interested in participating in the transmission and routing process. If the cluster node does not receive any acknowledgment from some of the nodes present in a particular group, then it concludes that they are not ready to participate in the routing process. At this situation, the cluster head will learn the topology constraints in the group and try to choose paths using source routing method.

There are two different conditions will be handled by the cluster head here.

\section{Condition 1}

Let the destination node $\mathrm{D}$, present in a group $g_{i}$ and holds the Contention Access Period (CAP). At this situation, the cluster head learns the topology restraint, and broadcast NP-Message and wait for the acknowledgment to be received. Once it gets the acknowledgment it will compute the path using source routing method. When the source route is computed then the cluster head unicasts the control message specifying the SCR-Source controlled routing to the nodes and also acknowledged the NP-Message. After the reception of the acknowledgement, original packet will be transmitted and the intermediate nodes just forward the packet specified in the route specified.

\section{Condition 2}

Let the destination node $D_{i}$ present in the group $g_{i}$, which does not hold the Contention Access Period (CAP).

At this condition, the cluster head allocates the contention access period for the specific group to which the destination node belongs asynchronously. The original data transfer will then be allowed through the group of the destination node. Once the data transfer is completed, then the access permission will be closed for the particular group.

\section{Group Controlling Algorithm}

In this destination address of all the packets are captured. After getting the destination address, the group address of the node has to be checked. Next, is to identify the group member which holds the Contention 
access period time slot. For the case when the destination matches, then it belongs to the same group which should have CAP. Identification of the group member which belongs to the same group is then followed. Next is to create NP message which should contains Destination address and group address. Then broadcast that NP message in the group and Set the broadcast timer. Here a node will not send acknowledgement if it already participates in a transmission or else it will send an acknowledgement. Then it stores the nodeid in the Packet transfer List. This Transmission process is done until the broadcast timer expires.

If it belongs to the same group, the next step is to find available path using topology details and neighbor lists. From the available path we have to select the optimum path from the packet transfer list where the nodes have to travel on the best path. Generate the Special Contention message which is special CAP allotted for the transmission which is asynchronous which has source controlled forwarding tag. Forward the packets through SCR using Unicast Transmission. After the transmission is completed the CAP has to be closed.

If it does not belongs to group holds CAP, Generate the Special Contention message which special CAP is allotted for the transmission which is asynchronous which has source controlled forwarding tag. Forward the packets through SCR using Unicast Transmission. After the transmission is completed the CAP has to be closed. The algorithm is depicted as follows:

1 int TotalGroupNumber;// Total number of sets groups) in a cluster.

2 Group G; //This is the list of all sets (groups).

$3|\mathrm{G}[\mathrm{i}]|=$ number of nodes in the group G[i];

$4 \mathrm{NB}=$ Neighbor matrix; // contains neighbor list of each node with bidirection //connection

5 Receive incoming packet $\mathrm{p}$.

NodeID $=\operatorname{Dest}(p) ; / /$ Destination address id of the packet

$\mathrm{Ga}=\mathrm{GA}($ NodeID);//Check for the group address of the Node.

$\mathrm{G}[\mathrm{i}]=\mathrm{CAP}(\mathrm{G}) ; / /$ Identify the group holds the CAP time slot.

$\varnothing(\mathrm{G}[\mathrm{i}] \times$ NodeID $) ; / /$ Check the destination is present in the group which has CAP.

If yes then // belongs to thesame group

$\mathrm{GM}=\Sigma(\mathrm{G}[\mathrm{i}]) ; / /$ Identify group members.

Create NP-Message.

NP-Messge $=\{$ NP, Destination Address, Group Address $\}$.

Broadcast NP-Message in the group.

Initialize broadcast timer Bt.

Until Bt->Expires

Ack=Receive NP-Acknowledgement. // here a node will not

//send acknowledgement if it

//already participates in a

//transmission

Store nodeid in the PT List. // PT - packet transfer list.

\section{Wait // end of until}

AvPath $=\quad$ From the PT_List identify available paths. //compute

$\mathrm{SCR}=\hat{\mathrm{O}}($ AvPath);///select optimal path //paths using topology details and neighbor lists.

$\mathrm{SCM}=$ Generate Special Contention message; // special CAP

//allotment for the transmission which is asynchronous

Unicast SCM to the list of nodes in SCR. which has source controlled forwarding tag.

Forward the packet through SCR.

Close CAP at transmission over.

Else // not belongs to the group holds CAP

$\mathrm{SCM}=$ Generate Special Contention message; // special CAP

//allotment for the transmission which is

$\mathrm{SCR}=\hat{\mathrm{O}}($ AvPath$) ; / /$ select optimal path

Unicast SCM to the list of nodes in SCR.
Forward the packet through SCR.

End

Close CAP at transmission over.

6 stop. 


\section{Results and Discussion}

The proposed clustering technique has been implemented using Network Simulator 2.0 (NS2) software. The NS2 affords a simulation environment in response to the network characteristics and condition. The channel model designed in NS2 is dependent and quickly responsive to the relative speed and environment. Extensive simulation analysis is performed so as to observe the effect of ICGM mechanism on a star-based topology. The performance of the network is also observed in terms of the network througput, the amount of energy consumed by the network nodes (energy consumption), latency (end-to-end delay), and the probability of successful transmission. The metrics higligted were also used in assessing the performance of slotted CSMA/CA MAC based protocol. The througput metric characterise the percentage of correctly received packets of information with respect to the total packets generated by the nodes in the network. However, the probability of successful transmission imitates the degree of reliability reached by the nodes in the network for effective transmission. In addition, the amount of packets sent from the application layer to the MAC sub-layer, regularized also, the overall network capacity. The IEEE 802.15.4 physical channel was open from interference with IEEE 802.11 networks operating at the similar frequency range that guarantees a reliable measurement process.It is accomplished by choosing Channel 26 for the IEEE 802.15.4 network and by means of a spectrum analyzer for examination of channel integrity.

The ICGM algorithm assigned four (4) time slots each to the different groupinorder tocharacterize a theoretical duration of 983.04 milli-seconds per group for asuper frameorder. It assumes equal group access duration for an equal number of nodes per group. Each of the sequencehas been carried out with different time slot and the data transfer is initiated in both inside-inside and outside-inside and inside-outside scenario.

Figure 2, we show the performance of the network with respect to localization delay in the network. The localization delay generated by the proposed approach also shows the difference generated by adopting the proposed ICGM mechanism. As can be observed from the graph, using our proposed mechanism (ICGM), the delay in seconds per unit of packet inter arrival time reduced drastically from $25 \mathrm{sec}$ in 10 packets inter arrival time to about $1 \mathrm{sec}$. Evidence is also seen in the other results with increased packets inter arrival time of up to 1000 . For the packet inter arrival time of 100 using ICGM, it is still $1 \mathrm{sec}$ and without using ICGM, it is 23 sec. Also, the graph further shows the decreased in delay per second of inter arrival time of packets in the network. It is observed that, localization delay decreases with increase in inter arrival time, which tends to $4 \mathrm{sec}$ for 1000 packets inter arrival time.

Figure 3 shows the performance of energy consumption of the proposed mechanism. The results were compared with an H-Name-e mechanism so as to see how our proposed mechanism performance with respect to energy consumption in the network. As can be seen from the Fig. 3, with increase in load in the network from about $0 \%$ to $60 \%$, there was not much difference in energy consumption, as it is a little drift from $15 \mathrm{MJ}$ to about $18 \mathrm{MJ}$. It became obvious when the increment of offered load increases from $60 \%$ to about $150 \%$, with a sharp increase from $18 \mathrm{MJ}$ to $36 \mathrm{MJ}$. Though the sharp increment of the energy usage is to aids in maintain quality of service (Reliability of the network nodes).

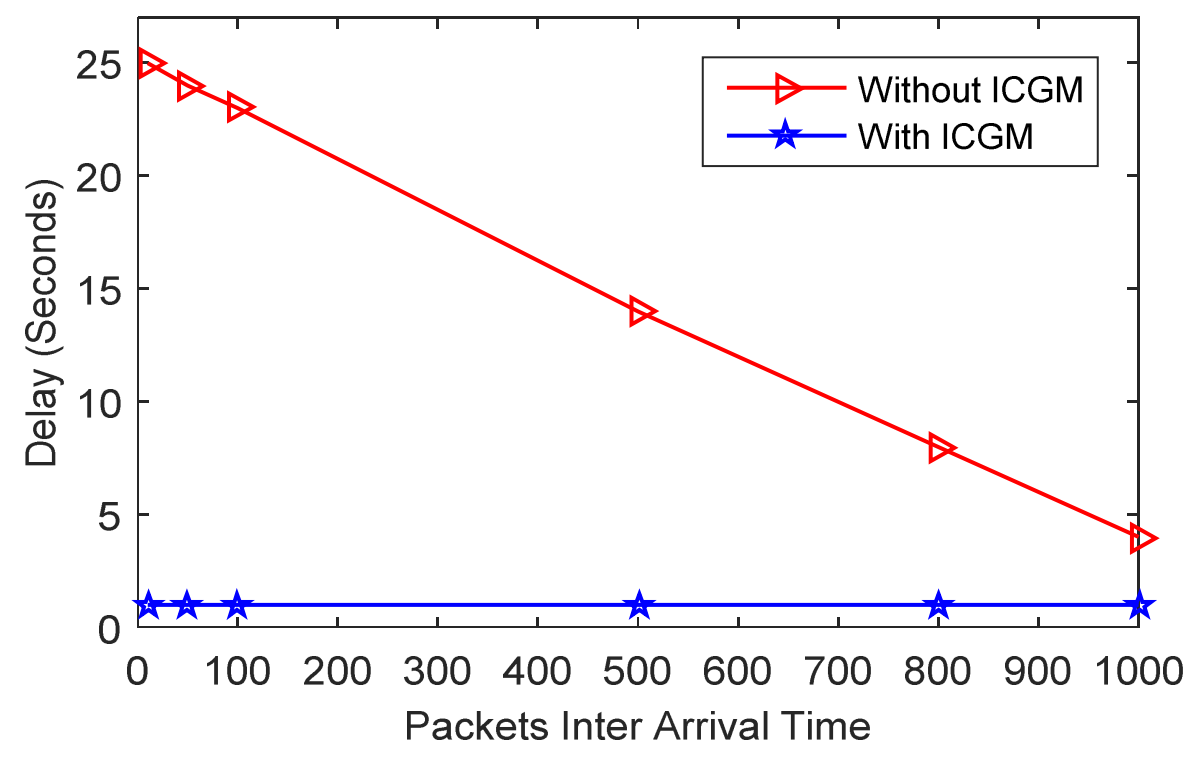

Fig. 2: A graph of localization delay 


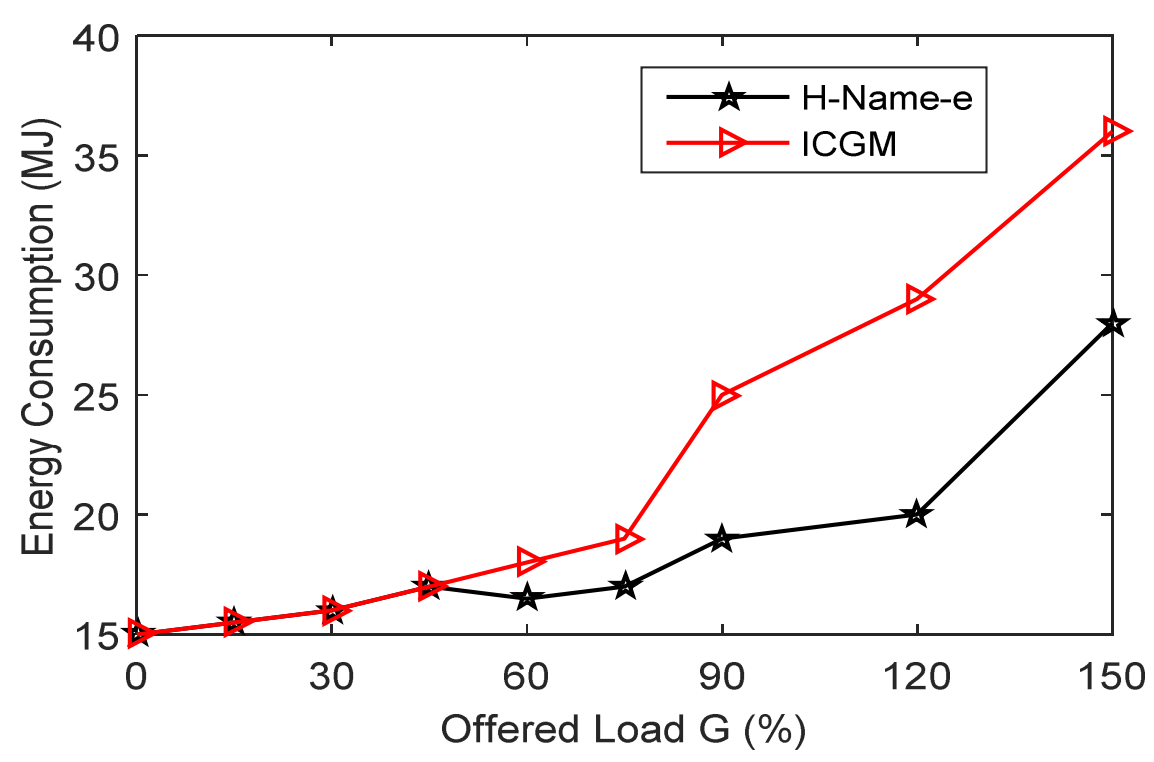

Fig. 3: A graph of energy consumption of nodes for different mechanisms

\section{Conclusion}

In this paper, we proposed an Inter-Cluster Grouping Mechanism (ICGM) to handles the problem of intercluster grouping and hidden node for the improvement of Quality of Service (QoS) factors in WSN. The performance of WSN degrades due to the occurrence of inter-cluster interference caused by different grouping mechanism as imposed in different clusters. The proposed mechanism (ICGM) overcome the problem of inter-cluster interference and increases the QoS in WSNs. From our simulation results it was seen that, the localization delay generated by the proposed approach tends to remain almost same value even as the packet inter arrival time was varied. Hence, the ICGM algorithms provide a better solution for solving the Inter cluster interference problem which affect greatly the quality of service in WSN as as a subset of wireless communication networks. In addition to the proposed mechanism, this work integrate IEEE 802.15.4/ZigBee protocols with out proposed mechanism (ICGM), which at presentis a promising communication technologies for wireless sensor networks and its applications. This integration leads to a simple mechanism for whichwireless sensor network nodes implementing ICGM will be fully transparent in terms of interoperability with the traditional WSN nodes. To conclude, the feasibility and effectiveness of the ICGM mechanism wasexecuted, experienced, authorized and established both in a real application scenario and in a dedicated test-bed, important to significant performance upgrading.This work can be extended to detect and mitigate the botnet attacks.

\section{Author's Contributions}

All authors equally participated in all experiments, coordinated the data-analysis and contributed to the writing of the manuscript.

\section{Ethics}

This article is original and contains unpublished material. The corresponding author confirms that all of the other authors have read and approved the manuscript and there are no ethical issues involved.

\section{References}

Farahani, S., 2008. ZigBee Wireless Networks and Transceivers. 1st Edn., Newnes, Amsterdam, ISBN-10: 008055847X, pp: 360.

Ghosh, R. and S. Basagni, 2008. Mitigating the impact of node mobility on ad hoc clustering. Wireless Comm. Mobile Comput., 8: 295-308. DOI: 10.1002/wcm.578

Hajiaghajani, F., 2012. HCMTT: Hybrid clustering for multi-target tracking in wireless sensor networks. Proceedings of the IEEE International Conference on Pervasive Computing and Communications Workshops, Mar. 19-23, IEEE Xplore Press, Lugano, pp: 889-894. DOI: 10.1109/PerComW.2012.6197637

Jurcik, 2007. The IEEE 802.15.4 OPNET simulation model: Reference guide v2.0 Technical Report, 2007. IPP-HURRAY.

Koscielnik, D., 2009. Influence of the hidden stations and the exposed station for the throughput of the LRWPAN. Proceedings of the IEEE International Symposium on Industrial Electronics, Jul. 5-8, IEEE Xplore Press, Seoul. DOI: 10.1109/ISIE.2009.5215718 
Koubaa, A., 2009. Improving quality-of-service in wireless sensor networks by mitigating hidden-node collisions. Ieee Trans. Industrial Informatics.

Mehta, R., 2012. Reforming clusters using C-LEACH in Wireless Sensor Networks. Proceedings of the IEEE International Conference on Computer Communication and Informatics, Jan. 10-12, IEEE Xplore Press, Coimbatore, pp: 1-4. DOI: 10.1109/ICCCI.2012.6158875

Pesovic, U., 2010. Hidden node avoidance mechanism for IEEE 802.15.4/ZIGBEE wireless sensor networks. PhD Thesis, University of Maribor.

Poonguzhali, P.K., 2012. Energy efficient realization of clustering patch routing protocol in wireless sensors network. Proceedings of the IEEE International Conference on Computer Communication and Informatics, Jan. 10-12, IEEE Xplore Press, Coimbatore, pp: 1-6. DOI: 10.1109/ICCCI.2012.6158872

Ranjani, S.S., 2012. Energy-efficient cluster based data aggregation for wireless sensor networks. Proceedings of the IEEE International Conference on Recent Advances in Computing and Software Systems, Apr. 25-27, IEEE Xplore Press, Chennai, pp: 174-176. DOI: 10.1109/RACSS.2012.6212719

Rohini, R. and R.K. Gnanamurthy, 2016. Performance analysis to improve quality of service using cluster based hidden node detection algorithm in wireless sensor networks. Intelligent Automation Soft Comput., Taylor Francis.
Rohini, R. and S. Ravi, 2017. Detection of residual nodes in wireless sensor networks by node weighting algorithm. Int. J. Printing, Packaging Allied Sci.

Sheu, S.T., Y.Y. Shih and W.T. Lee, 2009. CSMA/CF protocol 0for IEEE 802.15.4 WPANs. IEEE Trans. Vehicular Technol.

Shrestha, B., E. Hossain and S. Camorlinga, 2013. Hidden node collision mitigated CSMA/CA-based multihop wireless sensor networks. Proceedings of the EEE International Conference on Communications, Jun. 913, IEEE Xplore Press, Budapest, pp: 1570-1575. DOI: 10.1109/ICC.2013.6654738

Thampi, A., S. Armour, Z. Fan and D. Kaleshi, 2016. Mitigating inter-cluster interference on the uplink for a three-cell clustered cooperative network. Eurasip J. Wirel. Commun. Network. DOI: $10.1186 / \mathrm{s} 13638-016-0610-8$

Tobagi, F.A. and L. Kleinrock, 2009. Improving qualityof- service in wireless sensor networks by mitigating hidden-node collisions. IEEE Trans. Commun., 5: 299-313.

Yang, P. and B. Huang, 2008. QoS routing protocol based on link stability with dynamic delay prediction in MANET. Proceedings of the Pacific- Asia Workshop Computational Intelligence and Industrial Applications, Dec. 19-20, IEEE Xplore Press, Wuhan, pp: 515-518. DOI: 10.1109/PACIIA.2008.62 